\title{
MEASUREMENT OF INTER-STRAND CONTACT RESISTANCE IN EPOXY IMPREGNATED Nb3Sn RUTHERFORD CABLES
}

\author{
G. Ambrosio, E. Barzi, D. Chichili, L. Elementi, A.V. Zlobin \\ Fermi National Accelerator Laboratory \\ Batavia, IL, 60510, USA
}

\begin{abstract}
An apparatus for the measurement, under transverse pressure, of the inter-strand contact resistance in epoxy-impregnated $\mathrm{Nb}_{3} \mathrm{Sn}$ Rutherford cables has been recently assembled at Fermilab. Procedures have been developed to instrument and measure samples extracted from $\mathrm{Nb}_{3} \mathrm{Sn}$ coils. Samples were extracted from coils fabricated with the Wind-and-React and the React-and-Wind technology, both presently under development at Fermilab. A ceramic binder is used to improve the insulation and to simplify the fabrication of coils using the Wind-and-React technology. Synthetic oil is used to prevent sintering during the heat treatment of coils to be wound after reaction. In order to evaluate the effects of the ceramic binder and of the synthetic oil on the inter-strand resistance, measurements of samples extracted from coils were compared with measurements of cable stacks with varying characteristics. In this paper we describe the apparatus, the sample preparation, the measurement procedure, and the results of the first series of tests.
\end{abstract}

\section{INTRODUCTION}

In the last six years Fermilab has been developing $\mathrm{Nb}_{3} \mathrm{Sn}$ magnets for future hadron colliders, investigating both the Wind-and-React and the React-and-Wind technologies. The use of an inorganic ceramic binder (CTD 1002x) is the key ingredient of Fermilab's approach to the Wind-and-React technology. This binder helps to preserve the integrity of the insulation during winding and simplifies the coil fabrication by bonding all turns after curing [1]. On the other side, Mobil-1 synthetic oil is used to prevent bonding of strands during the heat treatment of cables to be used for the React-and-Wind technology. 

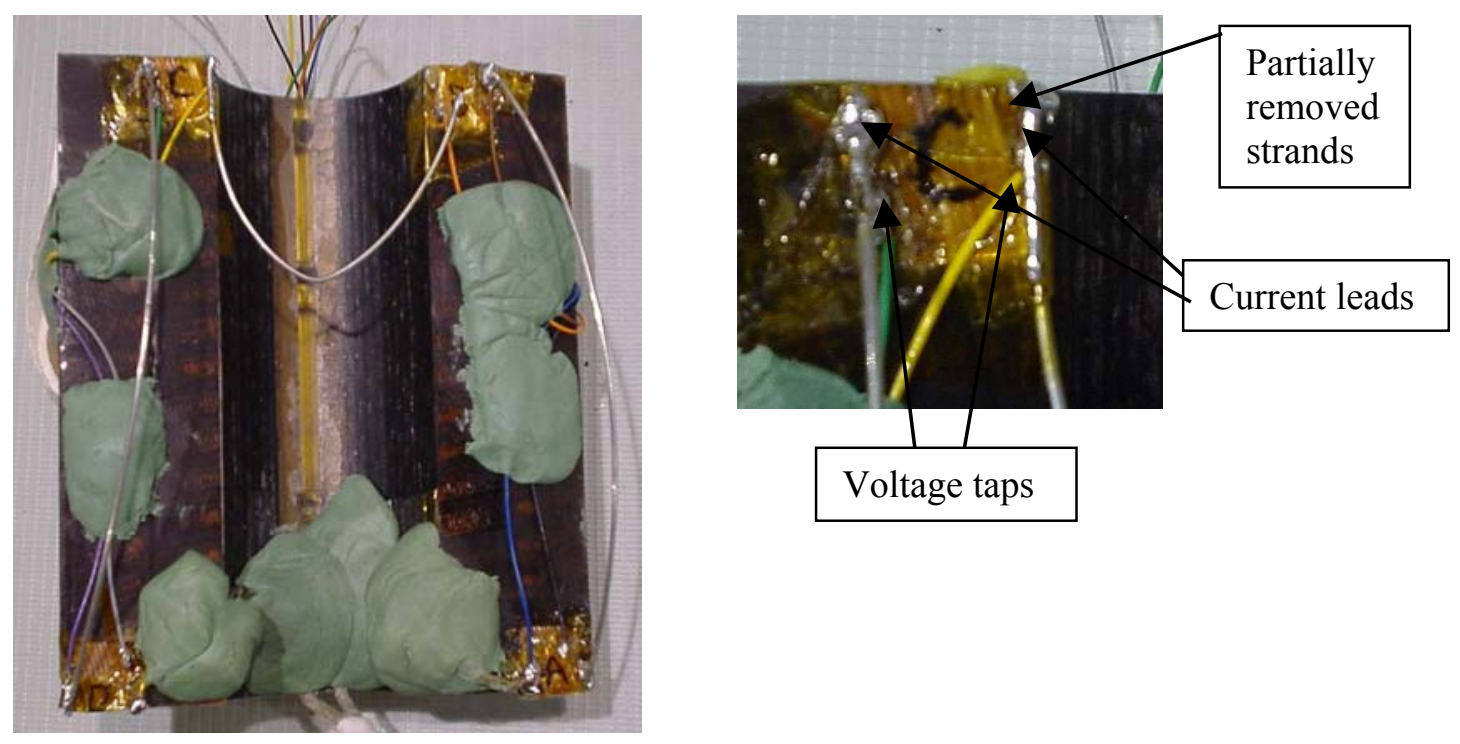

FIGURE 1. Sample B during instrumentation. On the right, detail of the instrumentation of a cable: a Kapton film covers all strands not connected to the leads; in the top-right corner, strands were partially removed to access a strand underneath.

The low values of AC loss and the ramp-rate dependence of the quench current seen during the tests of magnets fabricated with both technologies [2-3] raised concerns about possibly excessive values of inter-strand contact resistance (ICR) and its impact on cable stability.

At present measurements of ICR are performed in several laboratories using the electric method [4-6] or the calorimetric method [7], both well described in [8]. Measurements on $\mathrm{Nb}_{3} \mathrm{Sn}$ samples have been performed at CEA-Saclay [5] and at Twente University in collaboration with Ohio State University [7]. We decided to use the electric method (adapting an existing apparatus) and to focus our attention on testing both coil samples and cable stacks. Our goal is to measure the ICR in magnets and to compare the results with measurements on cable stacks, in order to understand the most important parameters, and to adjust the fabrication processes if required.

\section{APPARATUS DESCRIPTION}

The apparatus utilized for this measurement has been adapted from an existing apparatus used for measurement of elastic modules at cryogenic temperatures, consisting of a cryostat equipped with a $9000 \mathrm{Kg}$ load cell (double-acting oil cylinder filled by means a control valve). Current is brought to the sample, from the 100-A power supply, through two copper leads and NbTi superconducting strands. The current circulates through a shunt resistance, which allows for a precise current measurement.

The apparatus has been retrofitted with a multiplexer (Keithley 2001 switch system with a 7168 nanovolt scanner) and a digital voltmeter (Keithley 2182 nano-voltmeter). The system allows measurement at a fairly fast rate (about one sample every 50 milliseconds) of eight differential voltages with $7 \frac{1 / 2}{2}$ digits $(10 \mathrm{nV}$ at $100 \mathrm{mV}$ full scale) resolution. One of the eight channels of the system is dedicated to the measurement of the voltage drop across the shunt.

The multiplexer and the nano-voltmeter are remotely controlled through a PC with LabVIEW $^{\circledR}$ acquisition software. The pressure, read from the load cell, is recorded real- 

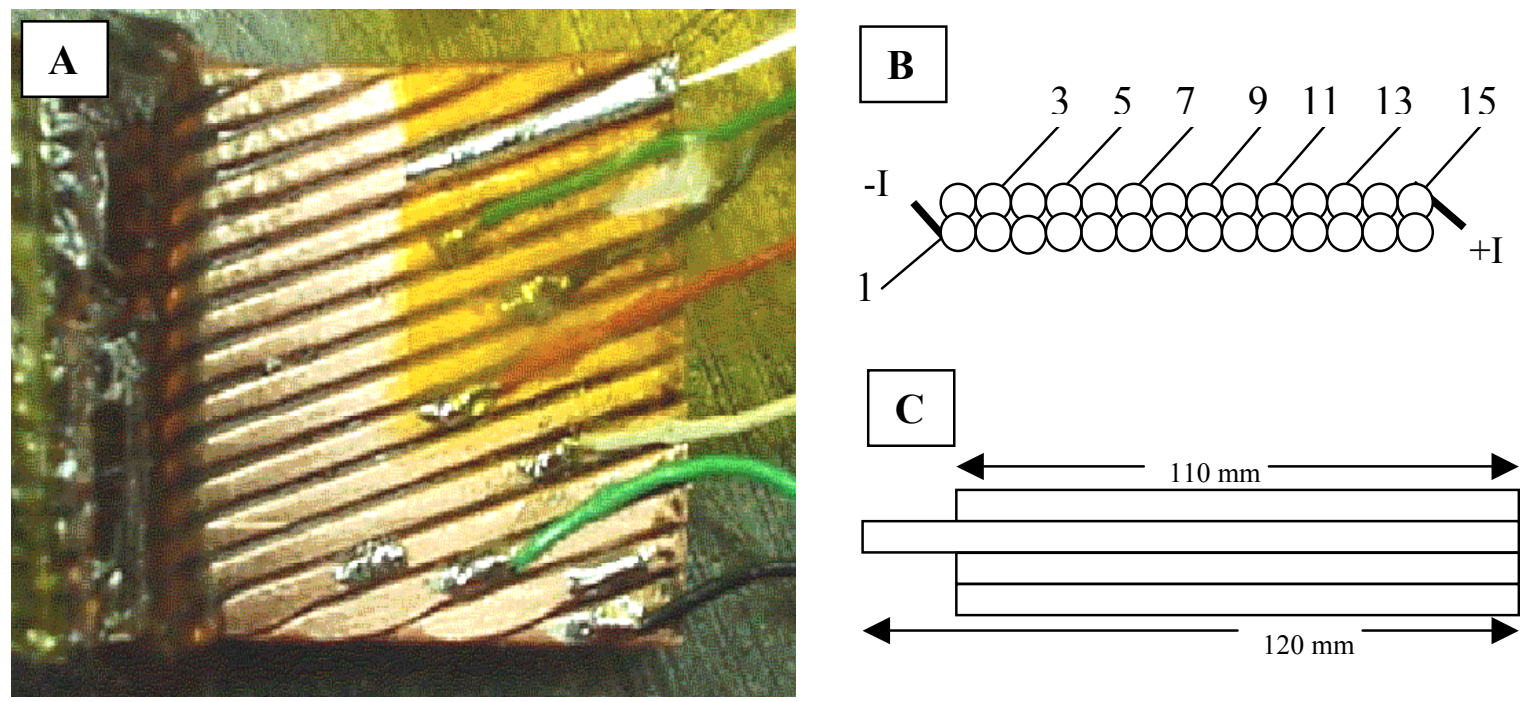

FIGURE 2. A) Instrumentation of sample F. The wire on the top is a current lead (note the long solder). A Kapton foil goes under the strand where the lead is soldered. B) Schematic of current leads and voltage taps. C) 4-cable stack for measurement under pressure.

time along with all other time and voltage data. The load is applied to the sample by manually tuning the control valve voltage, while the load cell measures the pressure. An upgrade is planned which will enable control of the pressure by means of a closed loop communicating with the acquisition program.

Test results did not show any significant effect of pressure on the ICR. Therefore, it was decided that testing under pressure would be applied to only some of the samples. It was also decided to increase the number of samples tested during each cooldown, by instrumenting four samples at a time with only two voltage taps per sample (referred to below as "multiple measurement").

Recently, in order to test several samples (five maximum) with seven voltage taps each, it was decided that a smaller cryostat with multiple probing ports should be implemented: each port is connected to a different sample and the user can choose where to tap the recorded voltage signal during the measuring process. In addition there is a liquid helium level sensor and 300-A current leads. An advantage of this new system (almost complete) is the light mass to be cooled at $4.2 \mathrm{~K}$ which allows for less helium consumption and may contribute to reducing the voltage drift during measurement. With the present apparatus all the liquid helium is spent in about two hours.

\section{SAMPLE PREPARATION AND DESCRIPTION}

Two kinds of samples have been tested: samples extracted from coils and samples made of cable stacks. The samples extracted from coils were prepared according to the following procedure. A section was cut from the coil straight section. This section was reduced to its final length (10 $\mathrm{mm}$ longer than a transposition pitch) by removing $25 \mathrm{~mm}$ from each end. These last two cuts were performed with a wet saw taking particular care not to "open" the ends of the sample (for instance some samples were cut after immersion in liquid nitrogen). The ends were carefully polished in order to avoid contacts among strands caused by residues from the cut. Epoxy and insulation were removed from a $10-\mathrm{mm}$ long section at the end of the cables to be tested (FIG. 1). In this area, current leads (NbTi wires) were soldered to two strands at the edges of the cables (for instance strands 1 and 15 
TABLE 1. Cable parameters.

\begin{tabular}{lccc}
\hline Cable & $28-1-\mathrm{No}$ & $28-1-\mathrm{ss}$ & $41-0.7-\mathrm{No}$ \\
\hline Strand diameter & 1 & 1 & 0.7 \\
Number of strands & 28 & 28 & 41 \\
Cable width $(\mathrm{mm})$ & 14.23 & 14.23 & 15.07 \\
Cable thickness: thin-thick edge $(\mathrm{mm})$ & $1.69-1.91$ & $1.69-1.91$ & 1.22 \\
Cable pitch length $(\mathrm{mm})$ & 110 & 110 & 110 \\
Stainless steel core thickness $(\mu \mathrm{m})$ & No core & 25 & No core \\
\hline
\end{tabular}

in Fig. 2). Because of the transposition angle, one of these strands is positioned on the bottom side of the cable. This necessitated removing six mm sections of some strands on the top side before soldering the lead (FIG. 1). Voltage taps were soldered on the strands to which the leads were soldered (both for single and multiple measurements) and on six additional strands (only for single measurement). Two techniques were alternatively used in order to prevent bonding of adjacent strands during soldering of leads or voltage taps: the target strand was isolated from the adjacent strand by a U-shaped thin layer of Kapton (FIG. 2), or the adjacent strands were protected by insulating varnish and masking tape.

Samples consisting of cable stacks were prepared following a similar procedure. Sections of cable were cut into two lengths: $20 \mathrm{~mm}$ longer than a transposition pitch (the "target cable", i.e. the cable to be measured) and five mm longer than a transposition pitch (the other cables). The ends of all cables were fused together by TIG welding, in order to avoid tin leakage during the heat treatment and to keep the ends of the strands together. The fused ends were filed to keep their thickness below the cable thickness (to avoid stress concentration points on the target cable during the compression of the cable stack before impregnation). The cables were stacked as shown in FIG. 2, heat treated, and then vacuum impregnated with CTD-101 epoxy under $7 \mathrm{MPa}$ pressure. The protruding end of the target cable was used for instrumentation. For this reason the impregnation was performed with the sample in a vertical position and was stopped when the epoxy covered all cables except the extra length of the target cable. Alternatively the end of the target cable was covered with mold-release varnish and silicon rubber (GE RTV 21) before impregnation. The instrumentation was set as in the coil samples, without the need to remove any part of the strands because both sides of the target cable were accessible.

Sample $G$ was assembled using pieces of reacted cable leftover from the winding of HFDB-02 [9]. The rest of the procedure was identical to the other cable stacks.

All cables tested were made of wires produced by Oxford Superconducting Technology using the Modified Jelly-Roll fabrication method. They had 54 sub-elements surrounded by a $\mathrm{Nb}$ barrier [10]. Cable parameters are presented in TABLE 1.

All samples are listed in TABLE 2. Samples tested in multiple measurements (e.g. A1 and A2) were instrumented with only two voltage taps, single samples (e.g. C and D) were instrumented with eight voltage taps. Samples A-F were made of 28-strand cable. None of the cables had a core with the exception of those used for sample F, which had a $25-\mu \mathrm{m}$ thick stainless steel core. All of the A and B samples consisted of two coil sections $10 \mathrm{~mm}$ longer than a transposition pitch. The coil belonged to HFDA-04 [11], a cos- $\theta$ magnet fabricated with the Wind-and-React technology using the ceramic binder and cable 28-1No. All cables on the coil midplane were tested. The layer of each sample is reported in TABLE 2. Sample C is the same sample A4, measured after being instrumented with eight voltage taps.

Sample D and each of the E samples were stacks of four cables each, reacted and impregnated together. The cables of sample E3 had the standard insulation of Fermilab cos- 
$\theta$ dipoles (ceramic tape and ceramic binder) [1]. The cables used for samples E1 and E2 were wrapped with a 25 - $\mu$ m-thick copper tape (50\% overlap), under the standard insulation, in order to reduce the amount of ceramic binder in contact with the cable. Sample D had no binder at all. Samples E1 and E3 were reacted under pressure provided by clamps. Samples D and E2 were reacted without pressure.

Samples G-I were made of 41-strand cable without core. Samples G and H consisted of sections of racetrack coils. Sample G was extracted from HFDB-01 [12] a racetrack magnet fabricated with the React-and-Wind technology. A small amount of Mobil-1 synthetic oil was applied during its cabling. The sample was not completely impregnated because of the premature curing of the epoxy used (828/NMA/DMP-30). This was the first sample we experimented with, it was longer than a transposition pitch, and the pusher bar used to apply pressure was shorter than a pitch. Sample H was extracted from a coil of HFDB-02 [3]. During the fabrication of this magnet, additional synthetic oil was added after cabling by means of oil impregnation under vacuum [3]. HFDB-02 coils were successfully impregnated with CTD-101 epoxy. Sample H was $120 \mathrm{~mm}$ long (10 mm longer than a transposition pitch). Some of the innermost and outermost turns were removed, and those exposed after this operation were tested.

Sample I was a three-cable stack made with leftovers reacted cable used to wind HFDB-02.

TABLE 2. Sample characteristics and measurement results

\begin{tabular}{|c|c|c|c|c|c|c|c|c|}
\hline "\# & Cable $^{\dagger}$ & $\begin{array}{c}\text { Sample } \\
\text { type }\end{array}$ & "Characteristics & $\begin{array}{l}\mathrm{R}_{\mathrm{TOT}} \\
(\mu \Omega)\end{array}$ & $\begin{array}{c}\mathrm{R}_{\mathrm{A}} \\
(\mu \Omega)\end{array}$ & $\begin{array}{c}\mathrm{R}_{\mathrm{C}} \\
(\mu \Omega)\end{array}$ & $\begin{array}{c}\text { Pressure } \\
\text { effect }\end{array}$ & Comments \\
\hline A1 & $28-1-\mathrm{No}$ & "HFDA-04 & Outer layer & 0.33 & & & & \\
\hline $\mathrm{A} 2$ & & & Inner layer & 6 & & & & \\
\hline A 3 & & & Inner layer & 0.49 & & & & \\
\hline A4 & & & Outer layer & 0.3 & & & & \\
\hline B1 & $28-1-\mathrm{No}$ & HFDA-04 & Inner layer & 26 & & & & Large error? \\
\hline B2 & & & Inner layer & 0.43 & & & & \\
\hline B3 & & & Outer layer & 0.31 & & & & \\
\hline $\mathrm{C}$ & $28-1-\mathrm{No}$ & HFDA-04 & Outer layer & 0.31 & 19 & 19 & & Was sample A4 \\
\hline $\mathrm{D}$ & 28-1-No & 4 cables & No binder $-\mathrm{W}$ press. & 0.06 & 1.2 & 10 & & Uneven $\mathrm{V}$ vs strand \\
\hline E1 & $28-1-\mathrm{No}$ & 4 cables & Cu tape - No press. & 0.24 & & & & \\
\hline E2 & & 4 cables & $\mathrm{Cu}$ tape $-\mathrm{W}$ press. & 0.14 & & & & \\
\hline E3 & & 4 cables & $\mathrm{W}$ binder - No press. & 0.21 & & & & \\
\hline $\mathrm{F}$ & 28-1-ss & 4 cables & $\mathrm{W}$ binder $-\mathrm{W}$ press. & 0.08 & 0.6 & $>>R_{A}$ & 0 & \\
\hline $\bar{G}$ & 41-0.7-No & "HFDB-01 & Poor impregnation & 2000 & & & $\sim 0$ & Short pusher \\
\hline $\mathrm{H} 1$ & 41-0.7-No & HFDB-02 & & 1700 & & & & \\
\hline $\mathrm{H} 2$ & & & & 8 & & & & \\
\hline H3 & & & & 120 & & & & \\
\hline $\mathrm{H} 4$ & & & & 30 & & & & \\
\hline $\mathrm{I}$ & 41-0.7-No & 3 cables & & 0.3 & 3 & $>>\mathrm{R}_{\mathrm{A}}$ & 0 & \\
\hline
\end{tabular}

$\uparrow$ Cable parameters are presented in TABLE 1.

* Sample types are: cable stacks ( 3 or 4 cables) or extracted from a magnet (i.e. HFDA-04).

\section{MEASUREMENT PROCEDURE}

Measurements were performed in boiling liquid helium at atmosferic pressure. The current was ramped from zero to the set value in 1-2 seconds, held for about a minute and then ramped down to zero. This procedure was repeated for several different current values, first increasing the set point by steps of $20 \mathrm{~A}$, up to $100 \mathrm{~A}$, and then decreasing it (i.e. 0, 20, 
$0,40,0, \ldots 100,0,80,0 \ldots)$. This full set of measurements was repeated one or more times in order to check reproducibility (sometimes with different current steps).

When possible (i.e. single sample set in the appropriate sample holder) the load cell was subsequently used to perform the same measurement under pressure (usually 10, 35 and $50 \mathrm{MPa}$ ). Sample $\mathrm{G}$ was measured only under 10 and $35 \mathrm{MPa}$.

The use of a stepped current profile was preferred to the slow constant increment used by other authors [6] because it allows for a more precise correction of the voltage offset at zero current. Typically this offset drifted by a few $\mu \mathrm{V}$ per minute during the first minutes after the cooldown, and by $2 \mu \mathrm{V}$ per hour after one hour (FIG. 3). When possible only data collected in the second hour after the cooldown were used for analysis, but sometimes it was necessary to use the data collected during the first hour (for instance when samples were tested at different pressures). The offset variation was cancelled by subtracting the average of the offset before and after measurement at the set current from the average of the measurements at the set current. Data collected during ramps and for a few seconds thereafter were discarded to avoid transient effects.

Measurements of voltage versus current were linearly interpolated to obtain the resistance between the strands connected to the leads $\left(\mathrm{R}_{\mathrm{TOT}}\right)$ and the resistance from the negative lead to each instrumented strand (only for samples measured singly).

In the analysis of samples with voltage taps on several strands, the program VIRCAB [13] was used to compute the adjacent $\left(\mathrm{R}_{\mathrm{A}}\right)$ and crossover resistance $\left(\mathrm{R}_{\mathrm{C}}\right)$ from the best fit of the results (FIG. 3). All fits were performed under the hypothesis of uniform distribution of both resistances within each sample.

\section{RESULTS}

The results are presented in TABLE 1 . The total resistance between the strands soldered to the current leads $\left(\mathrm{R}_{\mathrm{TOT}}\right)$ is reported for all samples. The adjacent $\left(\mathrm{R}_{\mathrm{A}}\right)$ and crossover $\left(\mathrm{R}_{\mathrm{C}}\right)$ resistance are reported only for the samples measured singly.
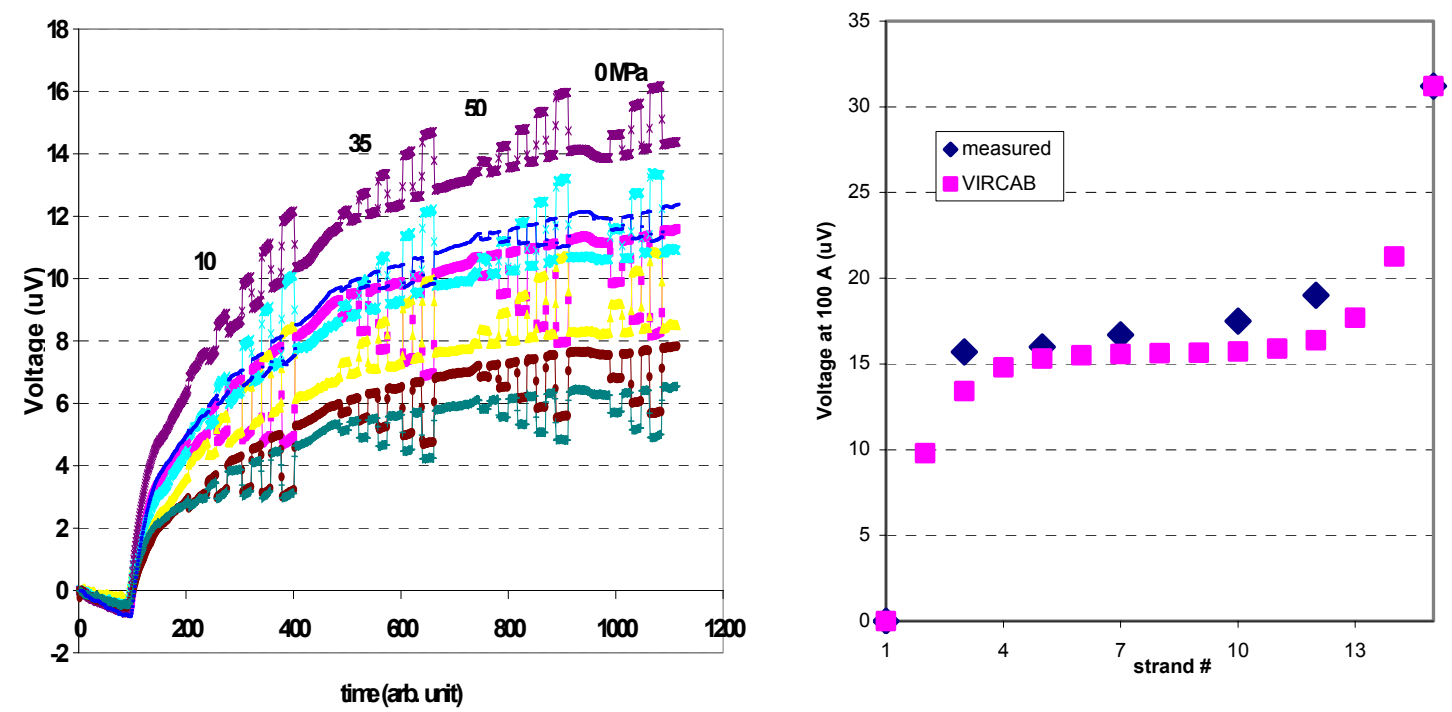

FIGURE 3. (Left) Voltages recorded during measurement of sample D at several pressures and currents. (Right) Voltage distribution in the strands of sample $\mathrm{C}$ and comparison with the distribution computed using VIRCAB. 
Each of the A and B samples showed values for total resistance lower than $0.5 \mu \Omega$ with one exception per sample. The result of sample B1 may be significantly in error because the solder to a current lead partially overlapped the solder to a voltage tap. Sample A4 was retested singularly. It showed the same total resistance previously measured (confirming the reproducibility of these measurements) and the voltage distribution among the strands (FIG. 3) revealed similar values for $\mathrm{R}_{\mathrm{A}}$ and $\mathrm{R}_{\mathrm{C}}(19 \mu \Omega)$.

Samples D-F had total resistances in the range 0.06 to $0.24 \mu \Omega$. Samples D and F, measured singularly, showed low values of $\mathrm{R}_{\mathrm{A}}$ (1.2 and $0.6 \mu \Omega$ respectively) and higher values of $R_{C}(10 \mu \Omega$ for sample $D$, and too high to be measured for sample $F$ with a core). These values are of the same order of magnitude of those reported in [5] and [7], but Devred [5] found lower values of $R_{C}$ with respect to $R_{A}$, in un-cored non-impregnated samples.

Sample $\mathrm{G}$ revealed a very high total resistance $(2000 \mu \Omega)$. It was measured singularly but we couldn't compute $\mathrm{R}_{\mathrm{A}}$ and $\mathrm{R}_{\mathrm{C}}$ because of the uneven voltage distribution among the strands. Sample $H$ showed values of total resistance in a very broad range (from 8 to 1700 $\mu \Omega)$. Sample I gave the lowest value of total resistance for the second set of cables $(0.3$ $\mu \Omega)$ with $3 \mu \Omega$ of $R_{A}$ and a significantly higher value for $R_{C}$.

None of the samples tested under pressure (F, G and I) showed significant change due to pressure. These findings are in agreement with measurements on non-impregnated $\mathrm{Nb}_{3} \mathrm{Sn}$ samples [5].

It should be noted that all cable stacks yielded results that were lower than those of coil samples fabricated with the same cable and technology. This may be an effect of possible differences in the amount of pressure during impregnation. All cables stacks were impregnated under $8 \mathrm{MPa}$ pressure. This is also the target value for the impregnation of coils fabricated with the Wind-and-React technology, but there are more uncertainties concerning the dimension of coils than cable stacks before impregnation, and all attempts to achieve the nominal dimensions aim at reaching values equal or lower than the target. In the case of coils fabricated with the React-and-Wind technology ( $G$ and $H$ ) the target pressure was less than $2 \mathrm{MPa}$. Both the large variation in results among samples extracted from a coil fabricated with this technology (H1 to $\mathrm{H} 4)$ and the lower value in the cable stack (I) could be explained by a non-uniform distribution of oil residues after heat treatment and/or by local differences of pressure during impregnation.

The results from coil samples are in good agreement with the AC loss measurement performed on HFDB-02 [14] and slightly lower than expected from measurement on HFDA-04 [2].

Devred [5] reported a change of slope in the V-I curve due to the double barrier $(\mathrm{Nb}$ and $\mathrm{Ta}$ ) of the strands he tested, because the $\mathrm{Nb}$ barrier contributed to the current transport below its critical field. The MJR strands we used have a single $\mathrm{Nb}$ barrier partially reacted. Therefore this effect, if present, should be significantly smaller. We are planning to retest some samples in a background field in order to evaluate this issue.

\section{CONCLUSIONS AND PLANS}

An apparatus for the measurement of the inter-strand contact resistance of $\mathrm{Nb}_{3} \mathrm{Sn}$ epoxy impregnated cables has been assembled at Fermilab. Procedures for the instrumentation of samples extracted from coils have been developed and used to obtain samples from $\mathrm{Nb}_{3} \mathrm{Sn}$ magnets fabricated with both the Wind-and-React and the React-andWind technology. The results presented, although preliminary because of the small number of samples measured, show that: 
o All samples extracted from coils fabricated with the Wind-and-React technology, using a ceramic binder, have acceptable values of inter-strand contact resistance.

o Samples extracted from coil fabricated with the React-and-Wind technology showed a large range of results, possibly caused by residues of synthetic oil, or by a low and non-uniform pressure on the coil during impregnation.

o All cable stacks gave results lower than those of coil samples fabricated with the same cable and technology, suggesting that the amount of pressure during impregnation may have an impact on the results.

o All samples tested under pressure (up to $50 \mathrm{MPa}$ ) showed no significant change.

A new apparatus is under assembly. It will allow the measurement of several samples with eight voltage taps during each cooldown. More tests will be performed in order to obtain more statistics and to evaluate the effect of synthetic oil and of pressure during impregnation. Some samples will be re-tested in a background field.

\section{ACKNOWLEDGMENTS}

This work is supported by the US Department of Energy.

\section{REFERENCES}

1. N. Andreev et al., " $\mathrm{Nb}_{3} \mathrm{Sn} \cos (\theta)$ dipole magnet HFDA-01 production report" Fermilab Technical Division report TD-00-069*.

2. S. Feher, et al., "HFDA-04 Test Summary" Fermilab Technical Division report TD-02-045*.

3. G. Ambrosio, et al.,"Fabrication and Test of a Racetrack Magnet Using Pre-Reacted $\mathrm{Nb}_{3} \mathrm{Sn}$ Cable" in IEEE Trans. Appl. Supercond., vol. 13, pp. 1284-1287.

4. D. Richter, et al., "DC Measurement of Electrical Contacts between Strands in Superconducting Cables for the LHC Main Magnets", CERN/CH-1211, 1996

5. Devred, et al., "Interstrand Resistance Measurements on $\mathrm{Nb}_{3} \mathrm{Sn}$ Rutherford-Type Cables", in IEEE Trans. Appl. Supercond., vol 9, pp 722-726

6. R. Soika, et al., "Inter-Strand Resistance Measurements in Cored Nb-Ti Rutherford Cables", in IEEE Trans. Appl. Supercond., vol 13, pp 2380-2383

7. M. Sumption et al., "AC loss of $\mathrm{Nb}_{3}$ Sn-based Rutherford cables with internally and externally added $\mathrm{Cu}$ " in IEEE Trans. Appl. Supercond., vol 13, pp 2376-2379

8. A.P. Verweij, Electrodynamics of Superconducting cables in Accelerator Magnets, Ph.D. dissertation, University of Twente, Enschede, The Netherlands, 1995.

9. G. Ambrosio, et al., "HFDB-02 $2^{\text {nd }}$ Racetrack Production Report", Fermilab Technical Division report TD-02-032*.

10. E. Barzi, et al., "Superconductor and Cable R\&D for High Field Accelerator Magnets at Fermilab", in IEEE Trans. Appl. Supercond., vol. 12, no. 1, pp. 1009-1013.

11. D. Chichili, et al., " $\mathrm{Nb}_{3} \mathrm{Sn}$ Cos (theta) Dipole Magnet, HFDA-04 Production Report”, Fermilab Technical Division report TD-02-025*.

12. G. Ambrosio, et al.,'Development and test of a $\mathrm{Nb}_{3} \mathrm{Sn}$ Racetrack magnet using the React and Wind technology"'in Advances in Cryogenic Engineering 47A, 2002, pp. 329-336.

13. A.P. Verweij, private communication.

14. S. Feher, et al., "HFDB-02 Test Summary" Fermilab Technical Division report TD-02-033*.

* Available on line at: http://www-td.fnal.gov/info/td_library.html 